\title{
Roles de género y turismo rural en el Valle Sagrado del Cusco
}

\section{[Gender roles and rural tourism in the Sacred Valley of Cusco]}

\author{
Molly Mellado (D) \\ Pontificia Universidad Católica del Perú
}

\begin{abstract}
Resumen
Esta investigación indaga sobre los cambios que trae la inserción e intensificación del turismo en el mundo rural y la influencia que ejerce sobre los roles de género. Para dicho fin, se realizó un estudio de caso con metodología cualitativa en el Valle Sagrado del Cusco en Perú y se descubrió que las dinámicas vinculadas al turismo rural han influido en los roles de género del ámbito familiar y el comunitario. En lo familiar, esto se produjo a través de la monetarización del trabajo doméstico, lo que vinculó a las mujeres al mercado laboral remunerado e incrementó la participación masculina en el hogar. En lo comunitario, los cambios se dan a partir del involucramiento de las mujeres en asociaciones turísticas y su vinculación con espacios urbanos. En este sentido, se busca analizar la magnitud del impacto del turismo y las posibilidades que abre en torno a la igualdad de género.
\end{abstract}

Palabras clave: roles de género, turismo, comunidades rurales, sociología rural

\begin{abstract}
This research explores the changes that the arrival and intensification of tourism brings to rural contexts and its influence on gender roles. A qualitative case study involving interviews and participant observation was conducted in the Sacred Valley of Cusco, Peru. We found that dynamics associated with rural tourism have influenced gender roles in both the family and community environments. On the family level, tourism drives the monetization of domestic work and the entry of women into the labor market, which in turn leads to a growing involvement of men in domestic tasks. On the community level, changes in roles are driven by the involvement of women in tourism associations and their growing links to urban spaces. Thus, the study provides an analysis of the magnitude of the impact of tourism on rural areas and the possibilities it generates in terms of gender equality.
\end{abstract}

Keywords: gender roles, tourism, rural communities, rural sociology

Contacto: La comunicación sobre este artículo debe ser enviada a Molly Mellado, email mmellado@pucp.pe

Financiamiento: La autora no declara fuentes de financiamiento. 


\section{INTRODUCCIÓN}

El turismo se ha intensificado a partir de la industrialización, la globalización, y la masificación de los medios de transporte. Debido a su impacto en las personas que viajan a otros lugares y para quienes las reciben, es necesario analizar los procesos turísticos desde distintas dimensiones y perspectivas. La mayoría de estudios sobre el turismo concentran su impacto en las grandes ciudades y el desarrollo potencial que puede tener para poblaciones urbanas. No obstante, pocas son las investigaciones que se han enfocado en la ruralidad y en las distintas características e impactos que tienen las dinámicas asociadas al turismo en los ámbitos rurales y urbanos. Los estudios de turismo rural en Latinoamérica se han realizado mayoritariamente en México y Brasil, existiendo muy pocas investigaciones desarrolladas en otros países latinoamericanos altamente turísticos como Perú. Los trabajos que se han realizado en Perú se han desarrollado principalmente con enfoques centrados en la gestión y la administración, y no existen suficientes estudios desde las ciencias sociales.

Existen importantes debates académicos en torno al turismo y sus efectos. En cuanto al turismo y la etnicidad algunos autores (van den Berghe, 1980) explican que el factor étnico-cultural y racial constituye uno de los principales factores de atracción para turistas en el turismo rural, puesto que se construye una imagen de un otro exotizado al cual se quiere visitar y comprender. De la misma manera, resaltan también las relaciones de poder existentes entre visitantes y visitados ya que, mientras los primeros están de vacaciones y realizando actividades de esparcimiento, los segundos se encuentran trabajando (Smith, 1992). Esto genera jerarquías y diferencias en los roles que desempeñan y en sus interacciones. Otros autores (Babb, 2019) resaltan la agencia de las personas que trabajan en turismo y destacan su participación activa en estos procesos. Aquí se debe prestar especial atención a las mujeres indígenas quienes, en muchos casos, emplean conscientemente aspectos de su identidad en las dinámicas del turismo con el fin de obtener ganancias y mejorar sus condiciones de vida y las de sus familias.

Respecto a la relación entre turismo rural y relaciones de género, la mayoría de las investigaciones han llegado a conclusiones similares respecto a la división sexual del trabajo en actividades vinculadas al turismo. Esta división sexual del trabajo refuerza los roles tradicionales de género, puesto que las mujeres tienden a desempeñarse en acciones relacionadas al hogar, la domesticidad, y el cuidado (Arzjani \& Rahiminezhad 2011; Babb 2012; Fuller \& Pérez, 2015). Asimismo, las actividades realizadas por las mujeres son generalmente menos valoradas que las que realizan los hombres, ya que ellos tienen más contacto con los turistas o dan la cara al público, a diferencia del trabajo femenino que, al darse en el hogar, es invisible o bien es invisibilizado (Flores-Ruíz et al., 2014; Fuller \& Pérez, 2015; Sparrer, 2003). Esto sucede principalmente porque se perciben las actividades vinculadas con el turismo rural comunitario como parte de sus labores de amas de casa y sus roles femeninos (Prados, 1999). 
Mellado, M. (2021). Roles de género y turismo rural en el Valle Sagrado del Cusco. Revista de Sociología, 36(2), 76-87. https://doi.org/10.5354/0719529X.2021.65605

Las mujeres rurales se encuentran vinculadas a los procesos productivos del turismo rural comunitario, pero no al acceso y gestión de recursos generados por dicha actividad (Fuller \& Pérez, 2015). Pese a ello, algunos investigadores son más optimistas respecto a la influencia del turismo rural para las mujeres, pues les da la posibilidad de insertarse laboralmente (Flores-Ruíz et al., 2014). Ellas valoran positivamente sus empleos y perciben de mejor manera el éxito subjetivo en base a su participación en la actividad turística (Diéguez et al., 2010; Ferguson, 2010). Finalmente, existe un consenso sobre la heterogeneidad de los casos de turismo rural y la idea de que el estudio de nuevos casos puede abrir las posibilidades para realizar nuevos descubrimientos en torno a esta temática.

A partir de lo señalado, este trabajo buscó analizar la influencia de las dinámicas socioeconómicas vinculadas al turismo rural comunitario en los roles de género de dos comunidades andinas en el Valle Sagrado del Cusco. A partir de las particularidades del caso estudiado, se buscó contribuir con los debates académicos en torno al turismo, la ruralidad, y los sistemas y roles de género en el mundo andino con enfoque en el desarrollo territorial rural y el género.

\section{MÉTODO}

Para esta investigación se optó por el uso de metodología cualitativa, pues esta permite profundizar en las características de la comunidad estudiada y obtener una mejor aproximación a las subjetividades de los individuos, sus percepciones, y valoraciones. Como técnica de producción de información se usaron entrevistas semiestructuradas, y observación participante.

Las entrevistas semiestructuradas se aplicaron a los casos seleccionados siguiendo una guía o pauta de preguntas y un orden establecido, pero también se las consideró maleables y se pudo moldear el curso de la entrevista o adaptar ciertas preguntas de acuerdo a las características de la persona entrevistada y las necesidades del momento. La observación consistió en contemplar sistemáticamente el desarrollo de las dinámicas socioeconómicas relacionadas al turismo rural en la comunidad y las relaciones entre hombres y mujeres, así como también los papeles que desempeñan cada uno de ellos. De esta manera, se capturaron los fenómenos y significados locales en las notas de campo. El carácter de participante de la observación se dio a partir del hecho que la investigadora estuvo desempeñando un rol en la comunidad y, pese a no estar inmersa en las dinámicas del turismo rural, fue observada por los pobladores e interactuó con ellos (Guber, 2001). El trabajo de campo se realizó en las comunidades de Amaru y Quello Quello, que están ubicadas en el distrito de Pisac de la región de Cusco, Perú.

Los informantes fueron seleccionados en función de que uno o más miembros de su hogar se dedicaran al turismo en jornada parcial o completa. Los informantes podían ser mujeres $\mathrm{u}$ hombres propietarios o empleados de un negocio vinculado al turismo (e.g., restaurante, agencia, hotel, etc.) o que ofrecieran servicios de alojamiento y/o alimentación 
Mellado, M. (2021). Roles de género y turismo rural en el Valle Sagrado del Cusco. Revista de Sociología, 36(2), 76-87. https://doi.org/10.5354/0719529X.2021.65605

en sus propias viviendas. De este modo, los informantes seleccionados están involucrados en las dinámicas socioeconómicas ligadas al turismo rural, por lo que suponen casos importantes en la investigación. Para la selección de los informantes se usó el muestreo por bola de nieve guiado por personas referidas por quienes fueron entrevistados previamente.

La producción de información se realizó en tres semanas no consecutivas entre los meses de julio y septiembre del año 2019. En ese periodo se pudieron realizar 17 entrevistas, de las cuales 13 fueron a trabajadores del turismo residentes en las comunidades mencionadas y 4 a actores clave para la investigación (i.e., dos guías de turismo, una funcionaria estatal y una representante de una ONG colaboradora). En total se desarrollaron
13 entrevistas, tres de las cuales fueron hechas a mujeres entre 18 y 29 años, cinco a mujeres de 30 años o más y cinco entrevistas fueron realizadas a hombres, todos ellos de 30 años o más. Se procuró que el número de entrevistas se distribuyera lo más equitativamente posible según sexo y grupo etéreo, pero esto fue complicado debido a la poca cantidad de hombres jóvenes dedicados al turismo. No se pudo entrevistar a ningún hombre entre 18 y 29 años, dado que la mayoría de ellos se encuentran trabajando fuera de la comunidad, realizando estudios, o no se dedican al turismo. El trabajo de campo implicó una mayor cantidad de entrevistadas mujeres que varones, pero esto se consideró válido ya que ellas se dedican más al turismo en Amaru y Quello Quello.

\section{RESULTADOS}

\section{Antecedentes y dinámicas}

La intensificación del turismo rural en los Andes peruanos parte de un interés creciente por la ruralidad, vinculado a una imagen construida de lo rural. Los turistas de espacios urbanos cada vez se sienten más atraídos hacia los espacios rurales por su medio ambiente y la naturaleza, y por las poblaciones rurales, a las que califican como exóticas. Así, las dinámicas del turismo rural comunitario en las comunidades son performadas por personas de la comunidad que se visten con vestimentas tradicionales que no usan cotidianamente, preparan almuerzos sumamente elaborados y enfatizan en sus tradiciones ancestrales.
La recepción de turistas se da principalmente a través de agencias de turismo ubicadas en la ciudad del Cusco o en Lima, pues el uso de internet en la comunidad es limitado y es difícil para los pobladores contactar a turistas $\mathrm{y}$ atraerlos hacia la comunidad. Por ello, la asociatividad es considerada un valor de suma importancia que les permite diversificar las fuerzas en la captación de turistas y tener más recursos en las actividades que desarrollan con ellos. La asociación La Tierra de los Yachaqs es la más grande y agrupa otras asociaciones. Esta asociación contó apoyo internacional y dispone de una oficina que funciona como agencia turística y permite captar turistas para las comunidades. 
Mellado, M. (2021). Roles de género y turismo rural en el Valle Sagrado del Cusco. Revista de Sociología, 36(2), 76-87. https://doi.org/10.5354/0719529X.2021.65605

De las seis asociaciones analizadas en este estudio, solo tres forman parte de La Tierra de los Yachaqs. Las otras trabajan de manera independiente y generan sus propios contactos con las agencias.

Las comunidades estudiadas se encuentran actualmente más vinculadas al turismo a partir de la recepción de turistas en sus hogares. Aunque están ligadas a esta actividad desde hace décadas a través de los circuitos tradicionales en los que trabajaban como porteadores, cocineros, tejedoras, u otros. En este sentido, el turismo rural comunitario no puede ser considerado una actividad nueva en el espacio rural o que genere un dinamismo que antes no existía en estos espacios. Contrariamente, el turismo rural comunitario actual es la intensificación de procesos que ya se venían dando hace mucho y la acentuación de viejos rasgos en un contexto de globalización acelerada. El turismo rural comunitario se debe entender como un fenómeno territorialmente situado, de relaciones sociales que se vinculan con elementos institucionales, productivos e identitarios propios de la ruralidad. Las formas de organización en asociaciones, la deliberación en asambleas y los cargos nominados a la usanza de la comunidad campesina reflejan una institucionalidad, organización social, y división del trabajo característica de las comunidades andinas. Asimismo, la identidad territorial y rural es importante para el desarrollo del turismo rural comunitario ya que implica procesos de memoria y reproducción de costumbres ancestrales, procesos de revalorización y asimilación a través de su demostración a otros.

\section{Roles de género}

Los roles de género de la comunidad se encuentran bastante definidos con tareas particulares que se asocian a lo masculino o lo femenino. Los varones se vinculan al trabajo agrícola y a trabajos estacionales en espacios urbanos, y las mujeres se encargan principalmente del hogar y del cuidado. Sin embargo, existen diferencias en relación a la edad. Las personas mayores de 50 se mantienen firmes en los roles ya establecidos, las personas más jóvenes señalan la importancia de distribuir las tareas con igualdad. Por ejemplo, los mayores señalan al varón como el jefe natural del hogar, pero los jóvenes señalan que no desean jerarquías en sus hogares y prefieren compartir la responsabilidad diciendo que ambos en la pareja son jefes o que ninguno lo es. De acuerdo a las entrevistas y a la observación participante, se pudo constatar que los roles y actividades tradicionalmente asociados a hombres y mujeres, se reproducen en los nuevos espacios que se forman. Aunque las asociaciones de turismo son relativamente nuevas y no se corresponden con instituciones antiguas o tradicionales -como la asamblea comunal, por ejemplo-la forma de participar, actuar, y decidir en las asociaciones se vincula a los roles de género tradicionales. Las asociaciones de turismo son protagonizadas por las mujeres debido a su estrecha relación con actividades vinculadas al trabajo doméstico. La participación de los hombres es relativamente baja y quienes participan se dedican generalmente a tocar música o a mostrar el trabajo agrícola a 
Mellado, M. (2021). Roles de género y turismo rural en el Valle Sagrado del Cusco. Revista de Sociología, 36(2), 76-87. https://doi.org/10.5354/0719529X.2021.65605

los turistas. Pese a la persistencia de los roles tradicionales de género, cuando hay mucho por hacer en el campo, las mujeres se involucran en agricultura; y cuando ellas trabajan en turismo, los hombres también participan del trabajo doméstico. El trabajo doméstico es valorado por la comunidad y por los hombres, quienes reconocen la importancia de mantener el hogar y cuidar a la familia. Esto se ha incrementado con la llegada del turismo pues cocinar, lavar y limpiar ahora implica también la obtención de ingresos. De esta forma, los hombres se han empezado a involucrar más que antes en el trabajo doméstico, ya que sus esposas en algunas ocasiones deben trasladarse a vender sus telares o se ocupan de las actividades con los turistas.

Finalmente, aunque los discursos tenían matices tradicionales, la observación participante mostró la agencia de la esposa en su relación de pareja y su habilidad para manejar situaciones, tomar decisiones en el hogar, y para persuadir a sus esposos de actuar de una u otra manera. En este sentido, lo que se menciona a nivel discursivo no necesariamente se condice con la práctica $\mathrm{y}$, si bien existen definiciones de las funciones de hombres y mujeres, son muchos los motivos que pueden llevar a que estas ideas no se lleven a cabo en la realidad y que las prácticas se den de forma diferenciada y compleja. De este modo, el turismo rural comunitario ha influido en los roles de género de las comunidades estudiadas en dos ejes principales: el ámbito familiar y el ámbito comunitario.

\section{Cambios en el ámbito familiar}

En el ámbito familiar, los cambios principales en los roles se han dado en relación a la monetarización de lo doméstico. Las actividades del turismo rural comunitario ahora se conciben como una extensión de las tareas domésticas aunque las actividades vinculadas al turismo no sean iguales al trabajo doméstico familiar. A esto se ha decidido llamarle monetarización de lo doméstico: el proceso mediante el cual tareas domésticas como cocinar y limpiar empiezan a generar ganancias económicas directas a partir del turismo rural. La monetarización de las actividades domésticas implica que las mujeres no participan únicamente del rol reproductivo, sino también en roles productivos tradicionalmente monopolizados por los varones. Las mujeres que obtienen y administran su propio dinero se sienten más independientes y tienen mayor autoestima, porque aportar económicamente al hogar es considerado muy valioso. Esto también ha generado cambios en los roles masculinos. Muchos de los varones que inicialmente estuvieron en contra de la instauración del turismo rural en la comunidad, acaban involucrándose en la actividad al ver su potencial económico y los ingresos que puede implicar para sus familias. La monetarización de un trabajo genera que el espacio doméstico sea más valorado por todos y que todos deban involucrarse en ello. Así, los hombres muchas veces terminan realizando actividades tradicionalmente consideradas como femeninas (e.g., lavar, cocinar, limpiar) en el marco de la recepción de turistas. 
Mellado, M. (2021). Roles de género y turismo rural en el Valle Sagrado del Cusco. Revista de Sociología, 36(2), 76-87. https://doi.org/10.5354/0719529X.2021.65605

La monetarización de lo doméstico aumenta el valor del trabajo en el hogar y a las personas que generalmente lo realizan: las mujeres. En este sentido, el sistema de prestigio categorizado por Anderson (1997) también se va modificando. Las actividades que antes eran muy poco valoradas empiezan a obtener reconocimiento de los demás. Eso se transforma en discursos que mujeres y hombres internalizan y asimilan. Ellas se sienten más valiosas y más útiles y su percepción en cuanto al rol y la agencia que desempeñan en sus familias va cambiando. Varias de las entrevistadas consideran que el hecho de aportar monetariamente en sus familias las pone en una situación de mayor equidad frente a sus parejas y les da mayor poder y agencia para tomar decisiones en sus hogares. Esto lleva a que se perciban como más activas, importantes y valiosas en sus familias y en su comunidad. Esta valoración también es forjada a partir del reconocimiento de sus esposos y de la comunidad, lo cual demuestra el carácter social y relacional de los roles de género, como se muestra en la Figura 1.

Figura 1. Mecanismos de Influencia de las Dinámicas del TRC en las Prácticas,

Discursos e Identidades de Género

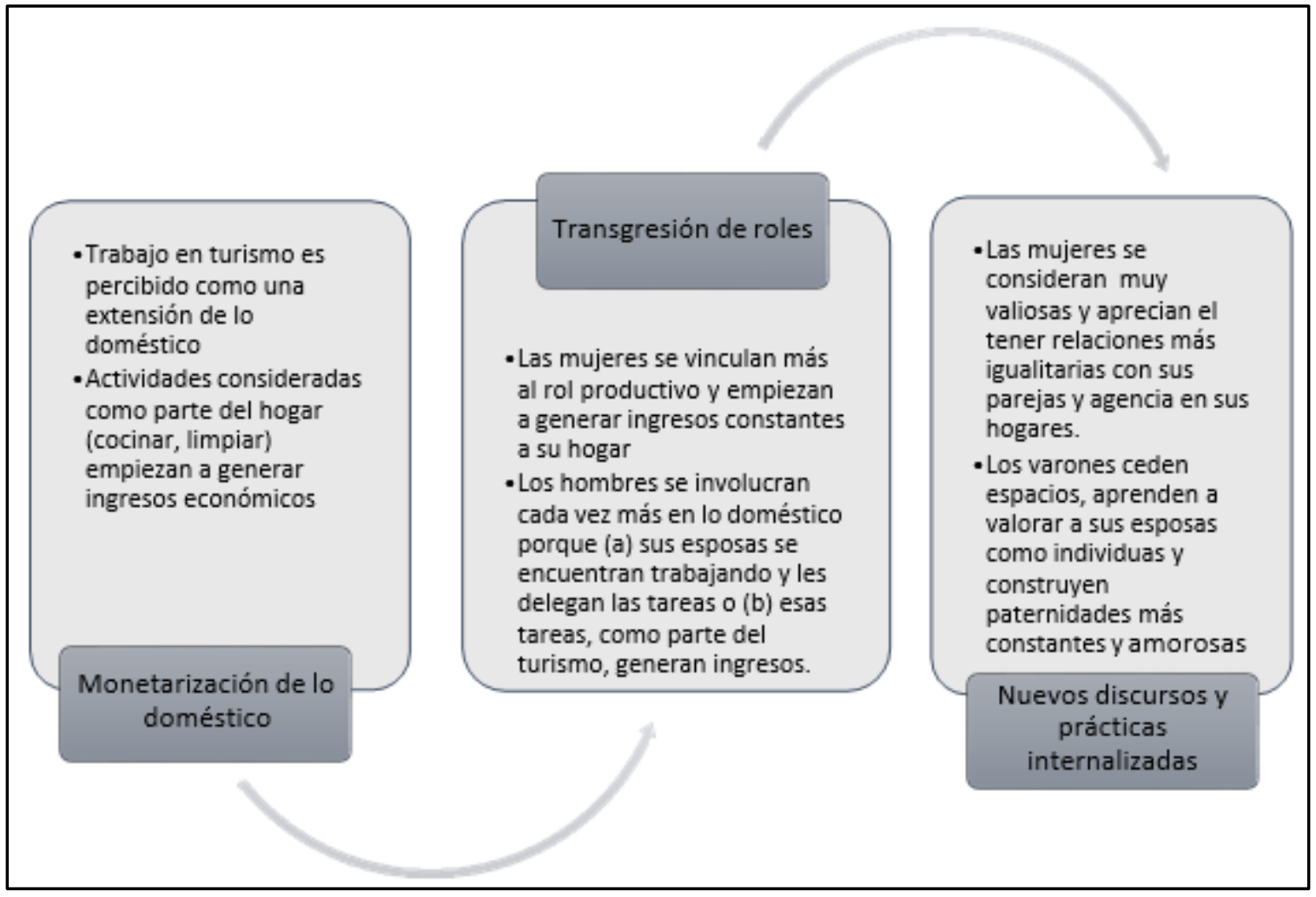


Mellado, M. (2021). Roles de género y turismo rural en el Valle Sagrado del Cusco. Revista de Sociología, 36(2), 76-87. https://doi.org/10.5354/0719529X.2021.65605

\section{Cambios en el ámbito comunitario}

El análisis de los cambios en el ámbito comunitario se hizo a partir de las transformaciones en los roles de género producto de la participación de ambos sexos en los espacios comunitarios, públicos y deliberativos asociados al poder y la toma de decisiones. Dado que las mujeres son tradicionalmente asociadas a la esfera doméstica, su participación y la presencia activa en espacios de la comunidad es algo transgresor. Sin embargo, el turismo sí ha tenido cierta influencia en la inserción de algunas mujeres a estos ámbitos y en la creación de nuevos espacios en los que también pueden participar.

La necesidad de trabajar en conjunto para conseguir más visitantes y mayores contactos por la demanda de la actividad ha generado que se funden y se consoliden las asociaciones de turismo como espacios de ejercicio de poder y de participación social y política. Estas asociaciones no están vinculadas a ningún órgano de política estatal o comunal, pero son ambientes en los que deben participar, organizar personas, y discutir ideas. La participación en asociaciones de turismo les da a las mujeres ciertas herramientas como liderazgo, habilidad para hablar en público, capacidad organizativa, entre otras; habilidades que les pueden servir para replicar esta participación en otros espacios políticos y de mayor poder. Otro elemento relevante es la posibilidad de las mujeres de construir redes de relaciones con otras mujeres. La existencia de asociaciones y organizaciones como las turísticas permite a las mujeres establecer conexiones y construir redes de relaciones con otras personas con quienes quizás tengan problemas, inquietudes, y sentimientos en común. De acuerdo a las entrevistadas, consideran a varias de las socias de sus organizaciones como amigas, por lo que disfrutan el tiempo que pasan juntas y logran compartir experiencias. El turismo y las asociaciones turísticas también implican vincularse con personas ajenas a la comunidad y de estar en constante diálogo con espacios y pobladores urbanos, lo cual implica retos y aprendizajes. Por ejemplo, algunas mujeres relatan que antes de su participación en turismo no sabían hablar castellano o no podían comunicarse muy bien en esa lengua, pero con su participación lograron aprender y tener acceso a cosas que solo podían acceder hablando castellano. Considerando la histórica invisibilización del quechua y otras lenguas andinas y amazónicas, es innegable que el hablar español implica un punto importante y de gran ayuda para estas mujeres y para alcanzar nuevas oportunidades.

La influencia del turismo en los roles en el ámbito comunitario se observa en los vínculos con el entorno urbano y las personas provenientes de él (e.g., visitantes, el mercado de Pisac, las agencias turísticas del Cusco, etc.). Esto contrasta con diagnósticos previos (de la Cadena, 1996), quien indicaban que las mujeres eran percibidas como más indias porque los hombres rurales tenían mayor conexión con los espacios urbanos que ellas. Actualmente las mujeres rurales indígenas son más exotizadas que los hombres y continúan siendo asociadas a la preservación de la comunidad y de las costumbres, pero el turismo las ha vinculado con el mundo urbano y esto ha acortado la brecha que las separaba de sus pares masculinos. La relación con espacios urbanos a través de aprender castellano, salir de la comunidad y vincularse con otros les ha dado más prestigio y estatus. Esto no 
Mellado, M. (2021). Roles de género y turismo rural en el Valle Sagrado del Cusco. Revista de Sociología, 36(2), 76-87. https://doi.org/10.5354/0719529X.2021.65605

ha implicado un proceso de occidentalización o blanqueamiento para ellas, pues trabajar en turismo en un espacio rural implica la preservación de costumbres ancestrales y la proyección de una identidad indígena a través de la vestimenta, la lengua y otras expresiones. De esta manera, el turismo es un vínculo con lo urbano y lo ajeno y también una conexión profunda con el pasado, los ancestros y la propia etnicidad.

La valía y consciencia de la importancia de las mujeres también se traduce en la agencia que tienen, tanto en el ámbito familiar como en el comunitario. Pese a la existencia de fuertes estructuras de poder, ellas deben ser reconocidas como sujetos políticos que luchan y negocian su posición en distintos espacios y que desarrollan estrategias para lograr sus objetivos. Siguiendo a Babb (2019), las mujeres andinas instrumentalizan estratégicamente su etnicidad y su condición de género para atraer turistas, pero lo hacen muy conscientes de la utilidad de ello para obtener ingresos y para desarrollarse como personas. En este sentido, las personas entrevistadas construyen una identidad en tránsito que condensa elementos de lo urbano moderno, aspiraciones de ingresar al sistema educativo y al mercado laboral capitalista, junto a un profundo respeto por sus tradiciones ancestrales y labores como la agricultura y la ganadería. Dado que el territorio está compuesto por elementos institucionales, productivos e identitarios, los entrevistados construyen sus roles y relaciones de género en base a instituciones como la familia, la asamblea comunal, los vínculos con su comunidad y otras formas de organización social propias del mundo rural andino. El ámbito productivo, a su vez, también ejerce una gran influencia sobre dicho tema y tiene un correlato en la división sexual del trabajo y vínculos fuertes entre las masculinidades rurales y el trabajo agrícola que son propias de esos espacios.

\section{Territorios y dinámicas}

El turismo y las dinámicas socioeconómicas asociadas a él también influyen en la identidad de género rural enmarcados en un contexto institucional y productivo. El turismo opera como una actividad económica que regula un modo distinto de producir en los hogares y forma nuevas instituciones y modos de organización social tomando como referencia instituciones propias de la ruralidad. Por ello, el territorio es una categoría importante para comprender la heterogeneidad de las poblaciones rurales y deconstruir lo rural y lo urbano como elementos dicotómicos para pasar a entenderlos como conceptos fluidos que tienen diversas áreas grises y muchas dinámicas intermedias. Lo rural ha sido históricamente tratado como opuesto a lo urbano usando categorías hechas para explicar procesos urbanos distintos. Por este motivo, aquí se procuró usar los conceptos familiar y comunitario como punto de partida para analizar los roles de género. En espacios pequeños, con pocos habitantes, y comunidades en las que todos se (re)conocen y están relacionados de alguna u otra manera, la separación entre lo privado y lo público es delgada o invisible. Por el contrario, lo familiar y lo comunitario están estrechamente vinculados, se influyen mutuamente y pueden mezclar lo privado y lo público y transitar en medio de ambas. De esta manera, se busca aplicar conceptos y categorías que estudien 
Mellado, M. (2021). Roles de género y turismo rural en el Valle Sagrado del Cusco. Revista de Sociología, 36(2), 76-87. https://doi.org/10.5354/0719529X.2021.65605

la ruralidad en sus propios términos y que den cuenta de la heterogeneidad de sus procesos y dinámicas y de la particularidad de las mismas.

Como se ha planteado aquí, los roles de género son los que diseñan las dinámicas socioeconómicas del turismo y la forma en cómo se distribuyen las tareas y responsabilidades. Los roles de género no son solo a aquellos vinculados con la división sexual del trabajo, sino también lo que se espera de hombres y mujeres. En este sentido, el turismo ha impactado en los discursos y prácticas de género en el ámbito familiar difuminando la distinción entre el rol productivo y reproductivo y que los hombres y mujeres tengan participación en ambos roles; y en el ámbito comunitario generando que las mujeres participen activamente en espacios comunales y que se vinculen más con el mundo urbano, acortando la brecha con sus pares masculinos. Si bien las estructuras y jerarquías de género siguen presentes en la comunidad y persisten muchas desigualdades y desventajas para las mujeres, las dinámicas socioeconómicas del turismo han impactado en los roles de género, generando matices y modificaciones rápidas y fluidas entre ellos.

\section{CONCLUSIONES}

Esta investigación tuvo por objetivo evaluar la influencia de las dinámicas socioeconómicas del turismo rural en los roles de género de dos comunidades del Valle Sagrado. A partir del trabajo de campo y el análisis de la información recogida, se pudo constatar que el turismo rural ha tenido una influencia considerable en hombres y mujeres en cuanto a los roles que desempeñan en sus hogares y en su comunidad.

En el ámbito familiar, esta influencia se da a través de la monetarización de lo doméstico, un proceso que implica que actividades domésticas tradicionalmente asociadas a lo femenino (e.g., cocinar, limpiar, lavar) empiecen a generar ganancias económicas directas a través del turismo rural y la recepción de turistas en los hogares de la comunidad. Este proceso genera que las labores realizadas por mujeres sean más valoradas, les permite lograr mayor autonomía económica y prestigio social frente a sus pares, e involucrarse directamente en el ámbito productivo del cual no siempre habían sido parte. Los hombres también han empezado a realizar actividades tradicionalmente consideradas como femeninas. Lo han hecho tanto para suplir la ausencia de sus esposas mientras ellas trabajaban en turismo, como para atender a los turistas que reciben. Así, se han modificado en parte los roles de género que desarrollaban en sus hogares.

En el ámbito comunitario, los roles de género se han visto afectados a partir de la participación de mujeres en las asociaciones de turismo, las redes sociales que han generado, y sus vínculos crecientes con el mundo urbano. Participar y, en algunos casos, liderar las asociaciones de turismo ha dado a las mujeres la posibilidad de ganar mayor voz en la comunidad que, actualmente, cuenta con más espacios de participación social y política en los cuales pueden involucrarse, de los cuales las mujeres habían sido tradicionalmente 
Mellado, M. (2021). Roles de género y turismo rural en el Valle Sagrado del Cusco. Revista de Sociología, 36(2), 76-87. https://doi.org/10.5354/0719529X.2021.65605

excluidas. Ser parte de asociaciones les ha permitido forjar amistades y vínculos con otras mujeres y compartir vivencias y experiencias más allá de sus hogares. A partir de su trabajo en turismo han experimentado la necesidad de aprender castellano y de estar en constante contacto con agencias turísticas, revendedores de artesanías y con un mundo urbano que es valorado y goza de mucho prestigio. En un contexto social de amplias desigualdades, lo urbano implica un capital simbólico que es de suma utilidad para ellas y al momento de disputar y negociar espacios con sus pares masculinos. Se pudo observar también que los roles y relaciones de género se encuentran en constante disputa y negociación. Dado que las comunidades conciben lo masculino y lo femenino de forma complementaria y dicotómica tanto en el discurso como en la práctica, lo que unos piensan y hacen impacta directamente en los otros. En los hogares y en la comunidad, hombres y mujeres negocian y disputan espacios, labores, y significados sobre lo que es y debe ser cada uno. Los roles de género se encuentran en constante negociación y tránsito, puesto que se enmarcan en un contexto de globalización y cambio social que también afecta la forma en cómo se desarrollan las relaciones de género.

Por último, este estudio resalta la agencia de las mujeres rurales y la importancia del territorio como categoría analítica. Las mujeres rurales viven en un contexto social de desigualdad de género y de machismo, pero demuestran ser activas en sus procesos de empoderamiento y en el desarrollo de sus vidas. En cuanto al territorio, es importante reconocer las características particulares en el mundo andino y rural para comprender los procesos sociales que se dan en estos espacios que, en muchas oportunidades, son analizados y observados con los lentes de la urbanidad, homogenizadores, o modernizadores. Las aproximaciones a la ruralidad deben y necesitan contar con categorías analíticas propias, que se ajusten a sus realidades y territorios para comprender a cabalidad los procesos en los que se encuentran inmersos.

\section{REFERENCIAS}

Anderson, J. (1997). Sistemas de género, redes de actores y una propuesta de formación. CEAAL.

Arzjani, Z., \& Rahiminezhad, V. (2011). Rural women and their roles in tourism. International Journal of Bio-Resource and Stress Management, 2(1), 1-4. https://bit.ly/3rSI8vu

Babb, F. E. (2012). Theorizing gender, race, and cultural tourism in Latin America: A view from Peru and Mexico. Latin
American Perspectives, 39(6), 36-50. https://doi.org/10.1177\%2F0094582X12454560

Babb, F. (2019). El lugar de las mujeres andinas. Retos para la antropología feminista descolonial. Instituto de Estudios Peruanos.

de la Cadena, M. (1996). Las mujeres son más indias: Etnicidad y género en una comunidad del Cusco. En P. Ruiz Bravo (Ed.), Detrás de la puerta: Hombres y mujeres en el Perú de hoy (pp. 181-202). PUCP. 
Mellado, M. (2021). Roles de género y turismo rural en el Valle Sagrado del Cusco. Revista de Sociología, 36(2), 76-87. https://doi.org/10.5354/0719529X.2021.65605

Diéguez, M., Gueimonde, A., \& Sinde, A. (2010). Éxito empresarial y género en turismo rural. Revista Tourism \& Management Studies, (6), 82-93. https://bit.ly/3IzvE1L

Ferguson, L. (2010). Turismo, igualdad de género y empoderamiento de las mujeres en Centroamérica. Papeles de Relaciones Ecosociales y Cambio Global, (111), 123133. https://bit.ly/3dF2UGS

Flores-Ruíz， D., Pulido-Fernández，J. I., \& Sánchez-Rivero, M. (2015). Women, rural development and tourism. Case analysis in rural regions of Andalusia. Spanish Journal of Rural Development, 6(1-2), 9-22. http://doi.org/10.5261/2015.GEN1.02

Fuller, N., \& Pérez, B. (2015). Turismo rural comunitario, género y desarrollo en comunidades campesinas e indígenas del sur del Perú. Quaderns, (31), 95-119. https://raco.cat/index.php/QuadernsICA/arti cle/view/308759/410725
Guber, R. (2001). La etnografía. Método, campo y reflexividad. Amorrortu.

Prados, M. (1999). Andalusian women and their participation in rural tourist trade. GeoJournal, 48(3), 253-258.

https://www.jstor.org/stable/41147378

Smith, V. L. (1992). Anfitriones e invitados: Antropología del turismo. Endymion.

Sparrer, M. (2003). Género y turismo rural. El ejemplo de la costa Coruñesa. Cuadernos de Turismo, 11, 181-197.

https://bit.ly/3IFc6ZO

van den Berghe, P. (1980). Tourism as ethnic relations: A case study of Cuzco, Peru. Ethnic and Racial Studies, 3(4), 375-392. https://doi.org/10.1080/01419870.1980.9993 312
Manuscrito recibido: 27-05-2021

Manuscrito aceptado: 30-11-2021 